\title{
Appendiceal mucinous cystadenoma
}

Fecha de recibido: 16-11-2010 • Fecha de aprobación: 15-12-2010

\begin{abstract}
Resumen. Informamos el caso de una paciente admitida al centro asistencial por dolor abdominal agudo. En base a los estudios clínicos y paraclínicos se intervino con el diagnóstico preoperatorio de apendicitis aguda no especificada. La laparotomía exploratoria mostró el apéndice con signos de inflamación y marcada distensión. El estudio histopatológico reporta un cistoadenoma mucinoso apendicular. Se revisa este tema por corresponder a una neoplasia quística poco frecuente del apéndice vermiforme, con poca probabilidad de diagnostico preoperatorio y con posibilidad de producir problemas serios, en oportunidades derivados del inapropiado manejo quirúrgico, como el pseudomixoma peritonei.
\end{abstract}

Palabras clave: mucocele, cistoadenoma, cistoadenocarcinoma, pseudomixoma peritoneal, apéndice.

\begin{abstract}
This is a report about a patient admitted to hospital because of acute abdominal pain. Based on the clinic and para-clinic studies it was carried out an intervention taking into consideration the preoperative diagnosis of non-specified acute appendicitis. Exploratory laparotomy showed the appendix as having signs of inflammation and a marked distension. The histopathological study reports an appendiceal mucinous cystadenoma. This issued is checked since it is a rare cystic neoplasm of vermiform appendix, with low probability of preoperative diagnosis and likely to cause serious problems which sometimes stem from an inadequate surgical management, like peritoneal pseudomyxoma.
\end{abstract}

Key words: cystadenoma, cystadenocarcinoma, mucocele, pseudomyxoma peritonei, appendix.

\section{INTRODUCCIÓN}

Las patologías neoplásicas del apéndice -tanto benignas como malignas- son infrecuentes a diferencia de las patologías inflamatorias.

Por su estructura microscópica en el apéndice cecal hay numerosas células caliciformes que generan abundante secreción exocrina de moco. Por esta razón la mayoría de las patologías neoplásicas epiteliales son mucoproductoras.

El término mucocele hace referencia a la dilatación de la luz apendicular por secreción de material mucoide viscoso. Puede ser causada tanto por procesos benignos como malignos ${ }^{(1)}$. De esta manera un mucocele es considerado como una obstrucción del apéndice debido a la excesiva acumulación de moco con dilatación anormal, pero el término "mucocele" no describe el potencial de agresividad o de comportamiento biológico y por tanto es un término descriptivo, pero no tiene el carácter diagnóstico o no corresponde a una entidad clínico-patológica definida.

\footnotetext{
* Médico Patólogo, Ciencias Básicas, Facultad de Salud, Universidad Surcolombiana. Correo electrónico: dpanqueba@gmail.com

** Cirujano General, Profesor Asistente, Departamento de Ciencias Clínicas, Facultad de Salud, Universidad Surcolombiana.

*** Estudiante Programa de Medicina, Facultad de Salud, Universidad Surcolombiana.
} 
Desde el punto de vista microscópico, el concepto de mucocele apendicular incluye diferentes patrones histopatológicos:

- Hiperplasia focal o difusa de la mucosa apendicular, en este caso el epitelio no muestra atipia nuclear.

- El cistoadenoma apendicular en el cual el epitelio es de carácter atípico o displásico, pero sin evidencia de invasión.

- Finalmente el cistoadenocarcinoma en donde además de la atipia epitelial hay infiltración por parte de células tumorales a las capas apendiculares subyacentes a la mucosa, con potencialidad de persistencia o diseminación.

- La extensión del proceso a la serosa peritoneal y su diseminación puede resultar en una ascitis mucinosa con siembras peritoneales conocida como pseudomixoma peritonei ${ }^{(2)}$.

\section{CASO CLÍNICO}

Paciente femenina de 53 años de edad quien consulta al servicio de urgencias por cuadro de dolor abdominal de inicio súbito, localizado en la fosa iliaca derecha, de 15 horas de evolución, sin asociarse a otra sintomatología gastrointestinal. Como antecedentes de importancia refiere laparotomías previas por cesárea e histerectomía. En los estudios paraclínicos, el hemograma reporta leve leucocitosis con neutrofilia y parcial de orina negativo para infección de vías urinarias (IVU). Al examen clínico y paraclínico no hay signos de respuesta inflamatoria, pero la valoración abdominal revelo hipersensibilidad franca en el flanco inferior derecho y fue intervenida con diagnostico preoperatorio de apendicitis aguda no especificada.

Se realiza laparotomía sobre cicatriz previa encontrando apéndice tensa, aumentada de tamaño, de $12 \mathrm{~cm}$ y $2,8 \mathrm{~cm}$ de diámetro en posición subcecal. La base tenía aspecto sano, en la región del ápex mostraba membranas fibrinopurulentas. En el anexo derecho se identifica una masa quística de origen ovárico de color violáceo de $6 \mathrm{~cm}$ de diámetro mayor, fija a la pared posterior del peritoneo parietal. El estudio histopatológico de esta lesión reporta quiste endometriosico del ovario, no relacionado con el hallazgo apendicular.
El reporte histopatológico describe un apéndice dilatado, de $9 \mathrm{~cm}$. de longitud, $2.7 \mathrm{~cm}$. de diámetro. La serosa es opaca, congestiva, hemorrágica con adherencia de membranas fibrinosas de color grisáceo.

Al corte hay dilatación marcada de la luz por material mucoide viscoso blanco. Se realizo amplio muestreo de la base de implantación del apéndice y de las áreas dilatadas. No se proceso la totalidad de la pieza quirúrgica.

En la figura 1, se observa la pared del apéndice con el epitelio mucoso. Se han borrado las criptas glandulares. El epitelio columnar muestra superposición y estratificación nuclear e irregularidad en el contorno y el tamaño de los núcleos. La cromatina es densa e irregularmente distribuida. Las características son de un epitelio neoplásico. No se evidencio invasión a las capas subyacentes. La lesión cumple con los criterios de un mucocele neoplásico no-invasivo y se clasifica como cistoadenoma con bajo grado de displasia.

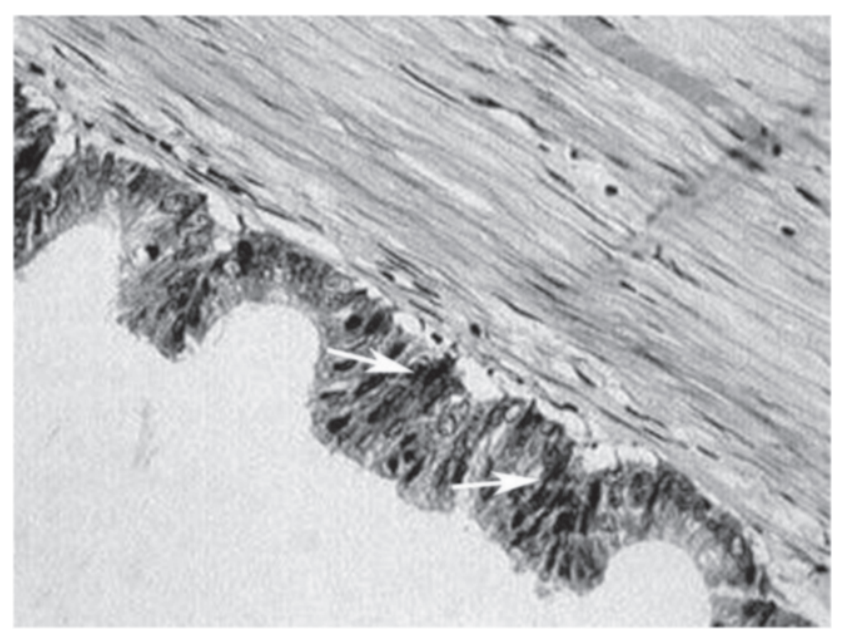

Figura 1. Apéndice. H-E. 400 x. Mucosa con pérdida de criptas. El epitelio columnar muestra atipia nuclear y estratificación (flechas). No hay invasión. Los hallazgos se catalogan como cistoadenoma mucinoso con displasia de bajo grado.

En el seguimiento post-operatorio se solicita tomografía de control a los 3 meses, que describe la cavidad peritoneal y región cecal sin anormalidades.

\section{DISCUSIÓN}

En una revisión de 1492 apendicetomías la prevalencia de patología neoplásica fue de 1,14\% 


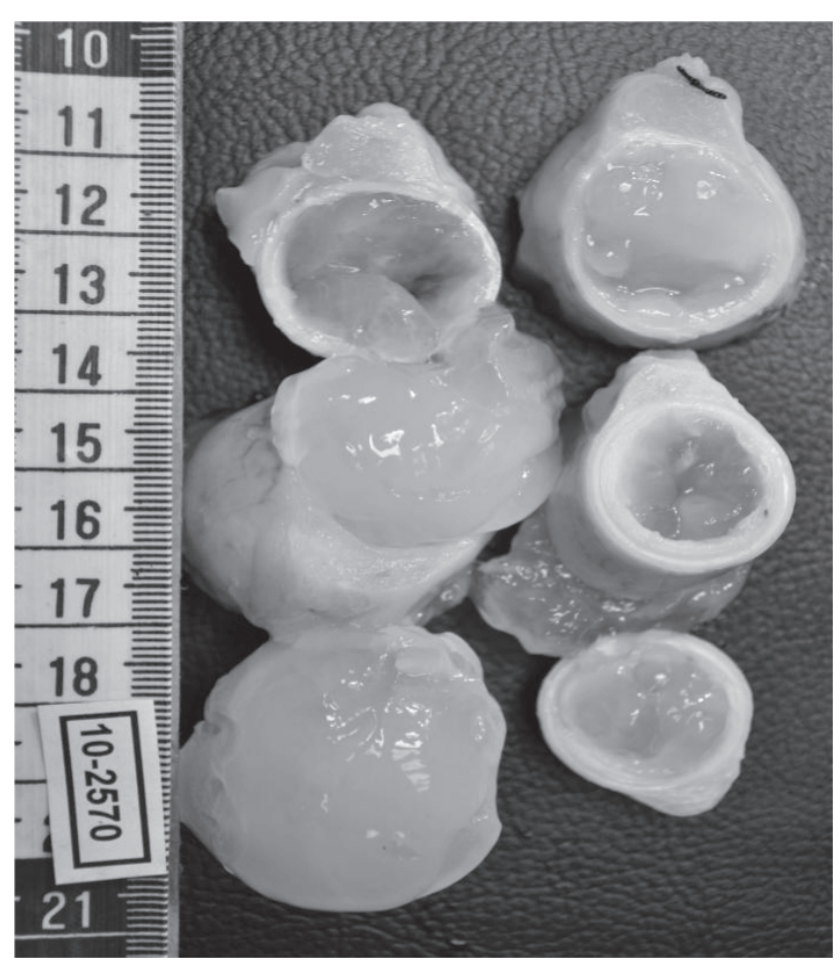

Figura 2. Vista macroscópica. Cistoadenoma mucinoso apendicular en cortes transversos. Muestra marcada dilatación luminal ocupada por material mucoide viscoso, blanco, adherente y engrosamiento mural.

(17 pacientes), encontrándose: carcinoides 0,54\% (8); cistoadenomas mucinosos $0,13 \%$ (2); adenocarcinoma $0,20 \%$ (3); cistoadenocarcinoma mucinoso $0,07 \%$ (1); enfermedad metastásica $0,07 \%$ (1) y pseudomixoma peritonei $0,07 \%(1)^{(3)}$.

En otra revisión de hallazgos histopatológicos de 2660 apendicetomías en Gales, se encontró la patología neoplásica en una proporción del 1,7\% (45 pacientes) de los cuales $0,52 \%$ (14 casos) correspondieron a tumores carcinoides; adenocarcinomas metastásicos $6+3$ primarios $0,39 \%$ (9); pólipos metaplásicos $0,23 \%$ (6); neurofibroma $0,04 \%$ (1) y cistoadenoma (neoplasia benigna) $0,6 \%(16)$. Es de resaltar en esta serie la frecuencia tan alta de cistoadenoma respecto a la incidencia de tumores carcinoide. La similar incidencia de cistoadenomas y carcinoides en este reporte constituye un hallazgo no usual en la literatura ${ }^{(4)}$.

Misdraji y cols. revisan 107 casos de neoplasias mucinosas apendiculares y las clasifica como:

- Neoplasias mucinosas de bajo grado 88 (82\%).

- Confinadas al apéndice: 39.
- Extrapendicular sin células: 10.

- Extrapendicular con células: 39.

- Adenocarcinomas mucinosos 16 (15\%).

- Compromiso extrapendicular 12.

- Inconcordantes $3(3 \%)$.

El impacto en la sobrevida del compromiso extraapendicular es claro. La mortalidad a $5 \mathrm{y}$ 10 años en las lesiones de bajo grado confinadas al apéndice es del 0\%; en las lesiones de bajo grado con compromiso peritoneal mostraron una mortalidad del $14 \%$ a 5 años y 55\% a 10 años. Las lesiones catalogadas como adenocarcinomas mucinosos registraron una mortalidad del 10\% a los 3 años y del $66 \%$ a los 5 años ${ }^{(5)}$.

Estas neoplasias se presentan generalmente en un promedio de edad cercano a los 60 años, afecta los dos sexos. Algunos estudios reportan mayor incidencia en mujeres y otros en hombres. En el 50\% de los casos es un hallazgo incidental y en el otro $50 \%$ es sintomático. En los sintomáticos en un $62 \%$ se diagnostica como apendicitis aguda manifestado predominantemente como dolor en cuadrante inferior derecho y seguido por sensación de masa ${ }^{(6)}$.

En un reporte de 31 casos de mucocele apendicular las formas más frecuentes de presentación clínica son: apendicitis aguda 45\% (14), tumores cecales $25 \%$ (8), líquido libre intrabdominal 9\% (3), obstrucción intestinal 3\% (1); dolor, pérdida de peso, anemia y otros en un 16\% $(5)^{(7)}$. Los diferentes tipos de lesiones mucinosas se muestran en la Tabla 1.(8).

\section{TRATAMIENTO}

La mayor parte de las neoplasias apendiculares son de tipo mucinoso. La incidencia de neoplasias de tipo intestinal -comunes en el colon y recto es escasa respecto a la variedad mucinosa.

No se recomienda el manejo laparoscópico del mucocele, dado que no es posible conocer su tipo histológico durante el procedimiento y que no se debe correr el riesgo de su ruptura transoperatoria.

\section{CONFINADAS AL APÉNDICE}

A diferencia de las neoplasias epiteliales de tipo intestinal, en las neoplasias mucinosas confinadas al apéndice mayores a $2 \mathrm{~cm}$. no se recomienda la hemicolectomía derecha, dado que hay compromiso ganglionar metastásico ocurre únicamente 
Tabla 1. Lesiones mucinosas del apéndice y peritoneo. Adaptada de Fenoglio-Preiser C, Noffsinger AE, Stemmermann GN, et al. 2008. Gastrointestinal Pathology: An Atlas and Text. New York, NY, Raven Press.

\section{Lesión}

Mucocele

Cistoadenoma mucinoso

(tumor mucinoso de

bajo grado)

Tumor mucinoso de potencial maligno incierto

Adenocarcinoma mucinoso

Cistoadenoma mucinoso con disección de moco

Pseudomixoma peritoneal

Mucinosis

Tumor mucinoso peritoneal de bajo grado

Adenocarcinoma peritoneal mucinoso

\section{Características}

Término macroscópico para denotar un apéndice dilatado por moco, secundario a una obstrucción neoplásica o no neoplásica.

Tumor benigno que a menudo rodea de manera circunferencialmente el lumen del apéndice. Puede presentarse con el aspecto de un mucocele. La lesión puede mostrar displasia de alto o de bajo grado.

Es un tumor mucinoso con áreas dudosas de carcinoma invasivo.

Es un carcinoma productor de mucina que típicamente se origina en un cistoadenoma mucinoso, invade la submucosa a través de la muscularis mucosae. Los focos invasivos están rodeados por una reacción desmoplasica y las células son citológicamente malignas.

Cistoadenoma de bajo o de alto grado con disección de moco hacia la pared pero sin traspasar la pared apendicular.

Presencia de colecciones de mucina en el abdomen y/o pelvis. Histológicamente puede mostrar mucinosis, tumor mucinoso de bajo grado, o un adenocarcinoma mucinoso.

Presencia de mucina en la cavidad peritoneal, incluyendo la superficie del apéndice. No hay células presentes en la mucina. Esta lesión usualmente complica un cistoadenoma mucinoso.

Estas lesiones también complican el cistoadenoma mucinoso. Los depósitos peritoneales mucinosos contienen células neoplásicas con los rasgos citológicos de un adenoma de bajo o de alto grado.

Los acumulos de moco contienen células citológicamente malignas. Estas células a menudo invaden los tejidos subyacentes. en el $4,2 \%$. No se ha demostrado que el compromiso en los ganglios regionales impacte en la sobrevida ${ }^{(9)}$.

Los adenocarcinomas intestinales tienden a diseminarse por vía linfática a los ganglios regionales, mientras que las neoplasias mucinosas su diseminación es por contigüidad, extendiéndose por la superficie peritoneal.

La conducta de realizar hemicolectomía derecha en las neoplasias mucinosas no tiene por tanto mucho sustento. El objetivo quirúrgico debe ser resecar el tumor con márgenes negativos. Por lo anterior el consenso actual en las neoplasias mucinosas del apéndice es insistir en un manejo más conservador y en el objetivo de obtener un margen negativo, se puede recurrir incluso a la cequectomia preservando el colon derecho y la función de la válvula ileocecal.

Acorde al reporte histopatológico del mucocele se puede optar por las siguientes conductas quirúrgicas:

- Mucocele con ruptura de la pared apendicular pero sin evidencia de células malignas en la superficie peritoneal (denominado mucinosis): se debe llevar a seguimiento tomográfico cada 6 meses por 5 años.

- La patología del mucocele reporta adenocarcinoma mucinoso confinado al apéndice: se debe llevar al paciente a un segundo tiempo 
quirúrgico mediante laparotomía amplia con exposición del abdomen y la pelvis, se deben revisar las superficies de los hemidiafragmas, transcavidad de los epiplones, ligamento de Treitz, receso paracolico izquierdo, receso rectovesical, rectouterino y toda la superficie del intestino delgado hasta la válvula ileocecal. Esto con el fin de prevenir la rápida diseminación del adenocarcinoma mucinoso que puede llegar a ser no detectable en la tomografía. Se toman muestras del omento mayor, menor y ganglios apendiculares e igualmente se administra quimioterapia hipertérmica intraperitoneal profiláctica.

- Mucocele con evidencia de células malignas que comprometen la superficie peritoneal: se debe llevar a un segundo tiempo quirúrgico, dando manejo como enfermedad diseminada, es decir cirugía de citoreducción y quimioterapia hipertérmica intraperitoneal.

Neoplasias con diseminación peritoneal en progresión: en el manejo de las neoplasias apendiculares mucinosas malignas con diseminación peritoneal estará indicado realizar cirugías citoreducturas que implican la resección de vísceras y peritonectomia a fin de borrar toda evidencia visible del tumor con la adyuvancia de quimioterapia intraperitoneal hipertérmica con el fin de eliminar la enfermedad microscópica. Se debe realizar disección de los ganglios apendiculares y si estos son positivos en este caso se recomendaría la hemicolectomía derecha ${ }^{(9)}$.

Los principales factores pronósticos de los mucoceles están dados principalmente por:

- Tipo histológico: la sobrevida de los pacientes llevados a cirugía citorreductora completa por lesiones de bajo grado es del $80 \%$ a 20 años, mientras que pacientes con lesiones de alto grado la sobrevida cae al $45 \%{ }^{(10)}$.

- Enfermedad confinada vs diseminada: En las neoplasias mucinosas de bajo grado confinadas al apéndice, el seguimiento a 6 años no evidencio recaídas tumorales. Mientras que neoplasias del mismo grado histológico con diseminación peritoneal mostraron una mortalidad del $55 \%$ a 5 años ${ }^{(10)}$.

\section{CONCLUSIONES}

El mucocele apendicular y en general las neoplasias epiteliales del apéndice son entida- des poco frecuentes, que raramente se identifican antes del manejo quirúrgico. Para la mayor parte de cirujanos puede ser una situación novedosa. Las serias consecuencias de no dar un manejo adecuado a la pieza quirúrgica -tratando de evitar la ruptura intraoperatoria- puede convertir una patología de manejo locorregional (ejemplo el mucocele por cistoadenoma confinado al apéndice) en una enfermedad diseminada potencialmente letal.

\section{REFERENCIAS}

1. De Rezende, Pereira, J.C., Trugilho, J.C., Sarmat, A.A. 2004. Mucocele of the appendix. Surgery 136(5):1096-1097.

2. Dixit, A., Robertson, J.H., Mudan, S.S. et al. 2007. Appendiceal mucocele and pseudomixoma peritonei. World Journal of Gastroenterology 13(16):2381-2384.

3. Ma, K.W., Chia, N.H., Yeung, H.W. et al. 2010. If not appendicitis, then what else can it be? A retrospective review of 1492 appendectomies. Hong Kong Medical Journal 16(1):12-17.

4. Marudanayagam, R., Williams, G.T., Rees, B.I. 2006. Review of the pathological results of 2660 appendicectomy specimens. Journal Gastroenterology 41(8):745-749.

5. Misdraji, J., Yantiss, R., Graeme-Cook, F. et al. 2003. Appendiceal mucinous neoplasmas: A clinicopathological analysis of 107 cases. American Journal Surgical Pathology 27(8):1089-1103.

6. Chen, H.T., Lee, Y.T., Chou, A.S. et al. 2006. Primary appendiceal malignancy: A clinic pathologic study. Kaohsiung Journal Medical Science 22(12):618-625.

7. García Lozano, A., Vázquez Tarrago, A., Castro García, C. 2010. Mucocele of the appendix: Presentation of 31 cases. Cirugía Española 87(2):108-112.

8. Fenoglio-Preiser, C., Noffsinger, A.E., Stemmermann, G.N. et al. 2008. Gastrointestinal Pathology: An Atlas and Text. New York, NY, Raven Press.

9. Gonzalez, S., Sugarbaker, P.H. 2004. Right hemicolectomy does not confer a survival advantage in patients with mucinous carcinoma of the appendix and peritoneal seeding. British Journal of Surgery 91(3):304-311.

10. Sugarbaker, P.H. 2009. Epithelial appendiceal neoplasms. Cancer Journal 15(3):225-235. 

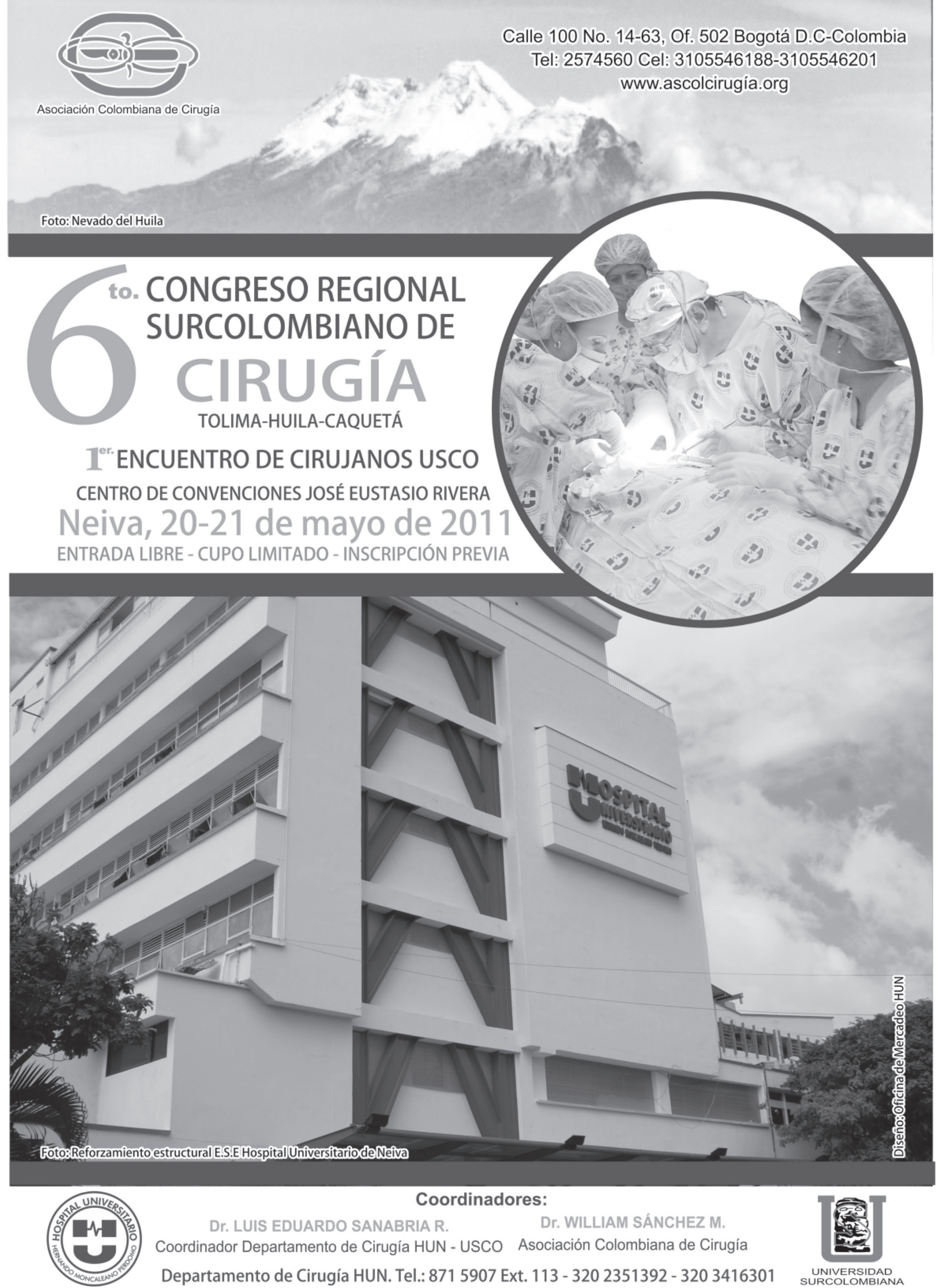

Coordinadores:

Dr. LUIS EDUARDO SANABRIA R.

Dr. WILLIAM SÁNCHEZ M. Coordinador Departamento de Cirugía HUN - USCO Asociación Colombiana de Cirugía Departamento de Cirugía HUN. Tel.: 8715907 Ext. 113 - 3202351392 - 3203416301

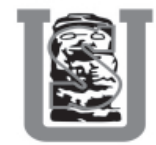

